\title{
Factors Determining Children's Private Health Insurance Enrolment and Healthcare Utilization Patterns: Evidence From the 2008 to 2011 Health Panel Data
}

\author{
Jawoon Shin ${ }^{1,2}$, Tae-Jin Lee ${ }^{2}$, Sung-il Cho ${ }^{2}$, Seung Ah Choe ${ }^{1,2}$ \\ ${ }^{1}$ Preventive Medicine Program, Graduate School of Public Health, Seoul National University, Seoul; '2 Graduate School of Public Health, Seoul National \\ University, Seoul, Korea
}

Objectives: Parental socioeconomic status (SES) exerts a substantial influence on children's health. The purpose of this study was to examine factors determining children's private health insurance (PHI) enrolment and children's healthcare utilization according to PHI coverage.

Methods: Korea Health Panel data from $2011(n=3085)$ was used to explore the factors determining PHI enrolment in children younger than 15 years of age. A logit model contained health status and SES variables for both children and parents. A fixed effects model identified factors influencing healthcare utilization in children aged 10 years or younger, using 2008 to 2011 panel data $(n=9084)$.

Results: The factors determining children's PHI enrolment included children's age and sex and parents' educational status, employment status, and household income quintile. PHI exerted a significant effect on outpatient cost, inpatient cost, and number of admissions. Number of outpatient visits and total length of stay were not affected by PHI status. The interaction between PHI and age group increased outpatient cost significantly.

Conclusions: Children's PHI enrolment was influenced by parents' SES, while healthcare utilization was affected by health and disability status. Therefore, the results of this study suggest disparities in healthcare utilization according to PHI enrollment.

Key words: Child, Parents, Health status, Insurance, Social class

\section{INTRODUCTION}

It is a well-known fact that, of numerous factors influencing health status, socioeconomic status (SES) exerts a considerable impact $[1,2]$. In particular, children's growth and health are

Received: October 5, 2015 Accepted: November 16, 2015

Corresponding author: Tae-Jin Lee, PhD

1 Gwanak-ro, Gwanak-gu, Seoul 08826, Korea

Tel: +82-2-880-2726, Fax: +82-2-745-9104

E-mail: tjlee@snu.ac.kr

This is an Open Access article distributed under the terms of the Creative Commons Attribution Non-Commercial License (http://creativecommons.org/licenses/bync/3.0/) which permits unrestricted non-commercial use, distribution, and reproduction in any medium, provided the original work is properly cited. strongly affected by environmental factors such as parenting deficiency and SES decline. The significance of these negative effects is considerable, as they persist throughout one's lifetime. Children from low-income households encounter problems of greater intensity in various areas, such as dietary habits, physical growth and development, inner maturity, health, behavior, and emotion, relative to those of other children, and children who are exposed to such problems are more likely to develop obesity and chronic disease in adulthood relative to those who are not [3]. Despite the importance of pediatric and adolescent healthcare, they involve a healthy population and are not noticeable in objective morbidity and mortality indicators; therefore, they are of low priority in the establishment of health polices [4-6]. 
In South Korea (hereafter Korea), although access to the health service has improved since the introduction of National Health Insurance (NHI) in 1989, many areas remain excluded from health insurance coverage, and $\mathrm{NHI}$ coverage constitutes $54 \%$ (as of 2012) of individual health expenditure, which is much lower relative to the Organization for Economic Cooperation and Development (OECD) average of $72 \%$. This insufficient $\mathrm{NHI}$ coverage has been an object of constant criticism, but raising statutory health insurance coverage to the OECD level is a challenge. Although the healthcare cost per capita is lower relative to the OECD average (USD 2275 as of 2013), national healthcare spending as a percentage of gross domestic product (GDP) increased rapidly from $3.6 \%$ in 1980 to $6.9 \%$ in 2013 [7]. For pediatric inpatients younger than 6 years of age, the copayment waiver policy for partial exemption from outof-pocket costs was established in January 2006. In this regard, Choi [8] compared healthcare utilization prior and subsequent to the introduction of the copayment waiver policy and reported a significant increase in pediatric healthcare utilization and expenditure between 2005 and 2006.

Under these circumstances, private health insurance (PHI) has played an important supplemental role in Korea. According to the 2009 Korea Health Panel data, the proportion of households that purchased PHI for at least one person was greater than $77 \%$, with the average number of $\mathrm{PHI}$ plans reported as 3.62 per household, exhibiting a constant upward trend [9].

Studies that have examined PHI plans thus far concern mainly adults and involve factors that influence insurance purchase, moral hazard, or adverse selection in healthcare utilization following PHI purchase [10-12]. Previous studies examining insurance enrolment factors for adults have indicated that socioeconomic variables, such as age, income level, marital status, educational level, residential area, occupation, chronic disease, and hospitalization experiences, were associated with insurance enrolment [13,14]. Park and Jeong [15] and Baek et al. [16] reported on PHI enrolment difficulties experienced by socially vulnerable population groups, such as the aged, individuals with little education and low income, and those who are not $\mathrm{NHI}$ beneficiaries, and examined the increase in the frequency of healthcare utilization following PHI enrolment. Most studies have found that SES is a determinant factor for PHI purchase decisions, and vulnerable social groups with poor health status face a high insurance enrolment threshold for cream-skimming and economic reasons [17-19].
No consensus has been reached with respect to whether $\mathrm{PHI}$ enrolment increases the frequency of enrollees' healthcare utilization. Yun [20] reported that healthcare utilization displayed age-dependent patterns, with PHI enrollees' in their 40s or older resorting to medical services less frequently relative to those in their 30s; in addition, $\mathrm{PHI}$ purchase did not increase the frequency of healthcare utilization, whereas cancer indemnity insurance enrolment resulted in significant increase in total healthcare and inpatient care expenditures, demonstrating the effect of PHI purchase on treatment intensity. Kang et al. [21] confirmed that while PHI purchase increased the frequency and duration of hospitalization, outpatient visits, and medical costs, it did not involve changes in inpatient medical costs. Kim [22] analyzed the short-term and long-term effects of indemnity insurance and reported that it increased the frequency of short-term outpatient visits and number of inpatient days, which reverted to baseline levels. Lee [23] inferred that PHI enrollees were more interested in cost-sharing types of insurance, as PHI purchase resulted in an increase in out-of-pocket health checkups and a decrease in $\mathrm{NHI}$ health checkups and vaccination rates. The inconsistent results in the healthcare utilization literature are attributable to the difficulties associated with PHI data acquisition and the need to control various individual health-related variables to acquire accurate statistics, as crosssectional analysis tends to underestimate enrollees' healthcare utilization. In addition, the performance of panel analysis is desirable, to rule out the influence of specific individual tendencies [24].

Pediatric healthcare utilization and insurance enrolment has not been studied extensively in Korea, and the scope of existing research is limited to taking stock of insurance enrolment [25]. According to some studies examining access to primary healthcare in other countries, insurance enrolment resulted in an increase in healthcare utilization in terms of hospital visit frequency, primary care physician enrolment, and prescribed medication [26-29].

Enrollees' moral hazard and adverse selection constitute two endogenous factors that are reflected in element analysis of insurance enrolment in adult members [30]. This results from information asymmetry in the insurance market, as suggested by Rothschild and Stiglitz [31], manifested as increased postenrolment healthcare utilization by individuals of high-risk groups with pre-existing conditions in need of intensive use of medical services, which is counteracted by insurance firms with a cream-skimming strategy to screen out those belonging to 
high-risk groups. In other words, unbiased analysis of the effects of insurance enrolment is almost impossible in studies involving adult subjects, because the patient groups in greatest need of medical care, such as the elderly (aged $\geq 60$ years) and those with chronic illnesses or disabilities, are excluded from statistics. However, preliminary health information is not available for children. Therefore, pediatric insurance enrollees lend themselves well to the analysis of adverse selection and creamskimming.

Against this background, this study aimed to examine the factors associated with children's PHI enrolment by analyzing not only child-specific individual (sex and age) and health-related variables (disability status and medication taken for 3 months or longer) but also adult-specific socioeconomic and health-related variables such as household income, educational level, employment status, marital (cohabitation) status, disability status, and chronic illness. A panel data analysis was performed to identify differences in outpatient and inpatient healthcare utilization patterns between children who had and had not enrolled in PHI.

\section{METHODS}

\section{Data Analysis}

Korea Health Panel data are held in a national database established by a consortium, which includes the Korea Institute for Health and Social Affairs and National Health Insurance Corporation, to produce basic data regarding medical expenditure, health status, health behaviors, and insurance enrolment for the country's inhabitants. The database includes PHI data collected via self-report questionnaires, which are used widely in $\mathrm{PHI}$-related studies. They also serve as the basis of this study.

The 2008-2011 annual Korea Health Panel datasets were used in this study. While most variables are presented as data for the entire year, $\mathrm{PHI}$ enrolment data are presented as datasets for the first and second halves of the year. The 2008 and 2010 databases consist of 6-month datasets, and those from the second half of the year were used in this study.

We took data for all PHI enrollees aged 10 years or younger from the Korea Health Panel 2008-2011 datasets and extracted their demographic (sex and age) and health-related (disability registration, medication taken for 3 months or longer) variables and their respective parents' socioeconomic (educational level, marital status, employment status, health insurance type, household income) and health-related (disability registration, chronic disease) variables. Model 1 included data for 3085 individuals from 1823 households with children younger than 15 years of age, extracted from 2011 Health Panel data from 5741 households and 17035 household members. Model 2 included 4-year panel data for 3233 subjects and 9084 corresponding observational values (i.e., an asymmetric panel with missing values).

\section{Variables}

As shown in Table 1, the variables used in this study concerned child-specific and parent-specific characteristics and consisted of sociodemographic and health-status variables, as suggested in a previous study. Because general characteristics of children's insurance enrolment depend largely on parental situations, only individual variables were used as child-specific characteristics and the remaining variables were considered identical to those of the parents.

With respect to child-specific characteristics, sex, age, health insurance type (NHI or Medical Aid), medication taken for 3 months or longer, and disability status were chosen as variables. Parent-specific characteristics included marital (cohabitation) status, educational level, disability status, employment

Table 1. Variable descriptions

\begin{tabular}{|c|c|}
\hline Variable & Definition \\
\hline \multicolumn{2}{|l|}{ Dependent } \\
\hline Private health insurance & $1=$ private insurance, $0=$ other \\
\hline \multicolumn{2}{|l|}{ Independent (child) } \\
\hline Sex & $1=$ male, $0=$ female \\
\hline Health insurance & $\begin{array}{c}1=\text { National Health Insurance } \\
0=\text { Medical Aid }\end{array}$ \\
\hline \multicolumn{2}{|c|}{ Medication taken for 3 months or longer $1=y e s, 0=$ no } \\
\hline Disability & $1=$ disabled, $0=$ other \\
\hline \multicolumn{2}{|l|}{ Independent (parent) } \\
\hline Marital (cohabitation status) & $\begin{array}{l}1=\text { unmarried, divorced, } \\
\text { or separated, } 0=\text { married }\end{array}$ \\
\hline \multirow[t]{3}{*}{ Educational level } & Elementary school or lower \\
\hline & Middle-high school \\
\hline & College or higher \\
\hline Disability & $1=$ disabled, $0=$ other \\
\hline Employment status & $1=$ employed, $0=$ other \\
\hline Chronic disease & $1=$ diseased, $0=$ other \\
\hline \multirow[t]{5}{*}{ Household income quintile } & $1=1$ st (lowest), $0=$ other \\
\hline & $1=2 n d, 0=$ other \\
\hline & $1=3 r d, 0=0$ ther \\
\hline & $1=4$ th, $0=$ other \\
\hline & $1=5$ th (highest), $0=$ other \\
\hline
\end{tabular}


status, chronic disease, and household income. The presence or absence of chronic disease and medication taken for 3 months or longer were used as health-status variables for adults and children, respectively. Medication taken for 3 months or longer, rather than chronic disease, was used for children, because chronic disease lacked relevance in children's health status. Household income levels were graded using quintiles.

\section{Study Models}

In this study, a 2011 cross-sectional analysis and a 20082011 time-series panel data analysis were performed to examine insurance enrolment-related changes in health expenditures. Accordingly, model 1, a logit model, was established in the 2011 cross-sectional analysis, to examine enrollee characteristics and PHI enrolment factors for children younger than 15 years of age.

$$
\begin{aligned}
& \operatorname{Pr}\left(Y_{i}=1\right)=P_{i}=1 /\left(1+\exp ^{-\left(b_{0}+b_{i} \times\right)}\right) \\
& \text { Logit }=\ln \left(P_{i} /\left(1-P_{i}\right)\right)
\end{aligned}
$$

\section{$\mathrm{Y}=\mathrm{PHI}$ enrolment dummy variable \\ $\mathrm{X}=$ sociodemographic variables for each child and parent pair}

In model 2, a fixed effects model adjusted for individual effects, panel data for 2008-2011 were used to analyze changes in healthcare utilization and expenditure for children aged 1 to 5 and 6 to 10 years according to PHI enrolment.

$$
Y_{i t}=X_{i t} \beta+u_{i}+e_{i t}
$$

$\mathrm{Y}=$ variable related to healthcare utilization: In (inpatient cost): number of admissions, number of inpatient days, In (outpatient medical cost): frequency of outpatient visits $\mathrm{X}=\mathrm{PHI}$ enrolment dummy variable + child's and parent's sociodemographic and economic variables

A panel fixed effects model consists of an explanatory variable $\left(X_{i t}\right)$, an error term $\left(u_{i}\right)$, which is constant over time and represents the characteristics of panel individuals, and a pure error term $\left(e_{i t}\right)$, which changes with each panel individual. While a random effect model hypothesizes the independence of error terms, a fixed effects model hypothesizes the non-independence of error terms. Therefore, the error term $\left(u_{i}\right)$ is the assumed parameter to be estimated. It is a general practice to use a fixed effects model because of the difficulties associated with hypothesizing the independence of various individual characteristics pertaining to the error terms and $\mathrm{PHI}$ enrolment in analyzing healthcare utilization rates.

The Hausman test was performed to determine the suitability of fixed and random effect models. In this test, the null hypothesis that independence exists between error terms and explanatory variables is established.

$$
H_{0}: \operatorname{cov}\left(\operatorname{Irexp} p_{i t} \text { or } x_{i t} a_{i}\right)=0
$$

In applying the Hausman test to the above hypothesis, the null hypothesis was rejected, revealing that the fixed effects model was more suitable for use relative to the random effect model [32]. The analysis was performed using Stata version 11.0 (Stata Corp., College Station, TX, USA).

\section{RESULTS}

\section{Subjects' General Characteristics According to Private Health Insurance Enrolment}

Table 2 presents the general characteristics of children younger than 15 years of age and the heads of their respective households ( $n=3085)$, according to the 2011 Health Panel data. Univariate analysis of characteristics according to PHI enrolment revealed that age, sex, and health insurance status differed significantly between the PHI and non-PHI groups, while the medication taken for 3 months or longer and disability status variables did not differ significantly between groups. With respect to the adult-specific variables, all six variables (cohabitation, education, disability, working, chronic disease, and household income) differed significantly between the PHI and non-PHI groups. Given that children's health insurance types largely coincide with those of their parents, sex and age group were the only child-specific features that differed significantly between the two groups.

\section{Factors Related to Children's Private Health Insurance Enrolment}

A logit model was used to examine PHI enrolment-related variables pertaining to child and parent pairs. Table 3 presents the result of the analysis. First, sex and age were identified as the children's individual sociodemographic variables that exerted a significant influence on insurance enrolment, while other variables, such as insurance type, did not show significant results. As for the variables pertaining to parents, educa- 
Table 2. General statistics for children and parents in 2011 ( $n=3085)$

\begin{tabular}{|c|c|c|c|c|}
\hline Variable & PHI & Non-PHI & Total & $p$-value \\
\hline \multicolumn{5}{|l|}{ Independent (child) } \\
\hline Age (y) & & & & $<0.001$ \\
\hline $1-5$ & $667(25.17)$ & 107 (24.59) & 774 (25.09) & \\
\hline $6-10$ & $920(34.71)$ & 109 (25.05) & $1026(33.35)$ & \\
\hline $10-15$ & $1063(40.11)$ & 219 (50.34) & $1282(41.55)$ & \\
\hline Sex & & & & 0.03 \\
\hline Male & 1395 (52.64) & 229 (47.36) & 1601 (51.90) & \\
\hline Female & $1255(47.36)$ & 206 (52.64) & $1484(48.10)$ & \\
\hline Health insurance & & & & $<0.001$ \\
\hline $\mathrm{NHI}$ & 2531 (95.51) & 376 (86.44) & 2907 (94.23) & \\
\hline Medical Aid & $119(4.49)$ & 59 (13.56) & $178(5.77)$ & \\
\hline Chronic medication & $126(4.75)$ & $13(2.99)$ & $139(4.51)$ & 0.07 \\
\hline Disability & $19(0.72)$ & $5(1.15)$ & $24(0.78)$ & 0.30 \\
\hline \multicolumn{5}{|l|}{ Independent (parent) } \\
\hline Cohabitation status & 2541 (95.89) & 376 (86.44) & 2917 (94.55) & $<0.001$ \\
\hline Educational level & & & & $<0.001$ \\
\hline Elementary or lower & 64 (2.42) & 48 (11.03) & 112 (3.63) & \\
\hline Middle-high school & $1150(43.3)$ & 196 (45.06) & 1346 (43.63) & \\
\hline College or higher & 1436 (54.19) & 191 (43.91) & 1627 (52.74) & \\
\hline Disability & 77 (2.91) & 34 (7.82) & $111(3.60)$ & $<0.001$ \\
\hline Employment status & 2533 (95.58) & 368 (84.60) & 2901 (94.04) & $<0.001$ \\
\hline Chronic disease & $1140(43.02)$ & $230(53.10)$ & 1371 (44.44) & $<0.001$ \\
\hline Household income & & & & $<0.001$ \\
\hline 1st (lowest) & $146(5.51)$ & 83 (19.08) & $229(7.42)$ & \\
\hline 2nd & 488 (18.82) & $104(23.91)$ & 592 (19.19) & \\
\hline $3 r d$ & $676(25.1)$ & 94 (21.61) & 770 (24.96) & \\
\hline 4th & 757 (28.57) & $87(20.0)$ & 844 (27.36) & \\
\hline 5th (highest) & $583(22.0)$ & $67(15.40)$ & $650(21.07)$ & \\
\hline Total & 2650 (85.9) & $435(14.1)$ & $3085(100)$ & \\
\hline
\end{tabular}

Values are presented as number (\%).

$\mathrm{PHI}$, private health insurance; NHI, National Health Insurance.

tional level, employment status, and household income quintile exerted a significant influence on insurance enrolment. The higher the educational and income levels, the higher the odds ratios, which implies that the likelihood of PHI enrolment increased with parental SES.

In contrast, the fact that neither of the children's health status variables (medication taken for 3 months or longer and disability status) yielded significant results implies that children's health status did not exert an influence on PHI purchase decisions, and the finding that parental disability status exerted a significant influence on PHI purchase indicated that disabilityinduced reductions in SES led to a lower rate of $\mathrm{PHI}$ enrolment for children. This was confirmed by the finding that children's
PHI enrolment rates decreased with household income.

\section{Panel Data Analysis of the Effects of Children's Pri- vate Health Insurance Enrolment on Healthcare Utilization}

The relationship between children's PHI enrolment and healthcare utilization rates was examined via a panel data regression analysis. To produce panel data, annual data from 2008 to 2011 were extracted for households with children aged 10 years or younger, and child-parent information was matched. The numbers of panel-based study subjects and 4-year observational values were 3325 and 9084, respectively. After assigning the children to 1 to 5 and 6 to 10 years age groups, a dum- 
Table 3. Factors determining purchase of children's private health insurance using a logit model, $2011(n=3085)$

\begin{tabular}{|c|c|c|c|}
\hline Private insurance & OR & $p>|z|$ & $95 \% \mathrm{CI}$ \\
\hline \multicolumn{4}{|l|}{ Sex } \\
\hline Male & 1.239 & 0.05 & $1.003,1.532$ \\
\hline Female & 1.000 & & \\
\hline \multicolumn{4}{|l|}{ Age (y) } \\
\hline $1-5$ & 1.110 & 0.44 & $0.851,1.447$ \\
\hline $6-10$ & 1.598 & $<0.001$ & $1.236,2.065$ \\
\hline $11-15$ & 1.000 & & \\
\hline \multicolumn{4}{|l|}{ Health insurance } \\
\hline National Health Insurance & 1.004 & 0.99 & $0.644,1.563$ \\
\hline Medical Aid & 1.000 & & \\
\hline \multicolumn{4}{|c|}{$\begin{array}{l}\text { Medication taken for } 3 \text { months or } \\
\text { longer }\end{array}$} \\
\hline Yes & 1.472 & 0.20 & $0.815,2.660$ \\
\hline No & 1.000 & & \\
\hline \multicolumn{4}{|l|}{ Disability (child) } \\
\hline Yes & 0.565 & 0.27 & $0.203,1.567$ \\
\hline No & 1.000 & & \\
\hline \multicolumn{4}{|l|}{ Cohabitation status (parent) } \\
\hline Separated in cohabitation & 1.494 & 0.06 & $0.984,2.268$ \\
\hline \multicolumn{4}{|l|}{ Educational level (parent) } \\
\hline Elementary or lower & 0.517 & 0.01 & $0.313,0.856$ \\
\hline Middle- high school & 1.037 & 0.76 & $0.819,1.314$ \\
\hline College or higher & 1.000 & & \\
\hline \multicolumn{4}{|l|}{ Disability (parent) } \\
\hline Yes & 0.561 & 0.01 & $0.352,0.895$ \\
\hline No & 1.000 & & \\
\hline \multicolumn{4}{|l|}{ Employment status (parent) } \\
\hline Employed & 1.795 & 0.009 & $1.161,2.776$ \\
\hline Unemployed & 1.000 & & \\
\hline \multicolumn{4}{|l|}{ Chronic disease (parent) } \\
\hline Yes & 0.863 & 0.19 & $0.692,1.077$ \\
\hline No & 1.000 & & \\
\hline \multicolumn{4}{|l|}{ Household income quintile } \\
\hline 1st (lowest) & 0.325 & $<0.001$ & $0.206,0.514$ \\
\hline 2nd & 0.639 & 0.01 & $0.448,0.914$ \\
\hline $3 r d$ & 0.865 & 0.41 & $0.615,1.219$ \\
\hline 4th & 1.009 & 0.96 & $0.717,1.421$ \\
\hline 5th (highest) & 1.000 & & \\
\hline
\end{tabular}

$\mathrm{OR}$, odds ratio; $\mathrm{Cl}$, confidence interval.

my variable was established to assign a value of 0 or 1 for Medical Aid or NHI, respectively. As asymmetric panel data, the data included in the analysis covered an average of approximately 2.3 years per panel group.

The children were assigned to 1 to 5 and 6 to 10 years age groups to reflect the effects of the copayment waiver policy on inpatients younger than 5 years of age. In addition, an interaction variable was established to identify differences in healthcare utilization and spending according to age group and $\mathrm{NHI}$ or Medical Aid group. Variables that do not change over time, such as sex, were excluded from the analysis in the fixed effects model. Moreover, infants younger than 1 year of age were integrated into the 1 to 5 years age group, as the availability of only the year of birth, without exact dates, created uncertainty.

In using a fixed effects model to obtain desirable estimates within a panel group, the presence or absence of autocorrelation should be established. The related test results revealed that first-order autocorrelation existed for the frequency of outpatient visits and number of inpatient days per year, excluding admission frequency, inpatient care costs, outpatient care costs, and total health spending. Therefore, if autocorrelation was exhibited, the effects of PHI on healthcare utilization were analyzed using the fixed effects model reflecting the first-order autocorrelation.

\section{Effects of children's private health insurance on outpatient care}

Table 4 shows the results of log-linear regression derived from the two independent variables: outpatient care costs and frequency of outpatient visits. The analysis produced the following findings:

First, the number of outpatient visits differed significantly according to age group in the fixed effects model. The 6 to 10 year age group showed approximately 5.5 fewer outpatient visits relative to the 1 to 5 years age group. Outpatient costs decreased with this reduction in outpatient visits. Second, in $\mathrm{PHI}$ enrollees, outpatient costs increased significantly, but the number of outpatient visits did not. This indicates that child's $\mathrm{PHI}$ enrolment resulted in higher outpatient care costs per visit without leading to an increase in the number of visits. Third, the interaction between insurance and age group led to a significant difference in outpatient costs between groups. Specifically, health expenditure increased by approximately 51\% when PHI-enrolled children moved from the 1 to 5 years age group to the 6 to 10 years age group. Given that outpatient costs are not affected by the copayment waiver policy, and the number of outpatient visits decreases with increasing age, increases in health expenditure for PHI-enrolled children were assumed to be considerable. 
Table 4. Fixed effects analysis of outpatients using 2008 to 2011 Health Panel data

\begin{tabular}{lcccc}
\hline & \multicolumn{2}{c}{ Log (outpatient cost) } & \multicolumn{1}{c}{ Outpatient visits } \\
\cline { 2 - 5 } & $\boldsymbol{\beta}$ & SE & $\boldsymbol{\beta}$ & SE \\
\hline Private health insurance & $0.2782^{* *}$ & 0.0862 & 0.481 & 0.4777 \\
Age group & $-1.5643^{* * *}$ & 0.1216 & $-5.5969^{* * *}$ & 0.6443 \\
National Health Insurance & $0.8943^{* * *}$ & 0.1318 & 1.5663 & 1.1166 \\
Chronic medication (child) & $0.6518^{* * *}$ & 0.1272 & $2.7527^{* *}$ & 0.8864 \\
Disability (child) & $1.6912^{* * *}$ & 0.2781 & $24.056^{* * *}$ & 1.3408 \\
Log (total household income) & $0.1502^{* * *}$ & 0.0420 & $0.8418^{* * *}$ & 0.2086 \\
Cohabitation status (parent) & $0.5628^{* * *}$ & 0.1345 & $2.4385^{*}$ & 1.1354 \\
Disability (parent) & $-0.4625^{* *}$ & 0.1456 & $-2.7963^{*}$ & 1.3283 \\
Educational level (parent) & $0.2816^{* * *}$ & 0.0478 & 0.7597 & 0.4217 \\
Employment status (parent) & 0.1172 & 0.1184 & 0.2004 & 1.0287 \\
Interaction' & $0.5138^{* * *}$ & 0.1267 & -0.2489 & 0.6601 \\
Constant & $7.6463^{* * *}$ & 0.3339 & $21.3039^{* * *}$ & 0.2477 \\
\hline SE, standard & & & & \\
\hline
\end{tabular}

SE, standard error.

${ }^{1}$ Interaction $=(\text { age group })^{*}($ private health insurance) .

${ }^{*} p<0.05,{ }^{* *} p<0.01,{ }^{* * *} p<0.001$.

Table 5. Fixed effects analysis of inpatients using 2008 to 2011 Health Panel data

\begin{tabular}{|c|c|c|c|c|c|c|}
\hline & \multicolumn{2}{|c|}{ Log (inpatient cost) } & \multicolumn{2}{|c|}{ No. of admission } & \multicolumn{2}{|c|}{ Total length of stay } \\
\hline & $\beta$ & SE & $\beta$ & SE & $\beta$ & SE \\
\hline Private health insurance & $0.390 * *$ & 0.1220 & $0.052^{*}$ & 0.0259 & 0.259 & 0.1867 \\
\hline National Health Insurance & $-0.407^{*}$ & 0.1866 & $-0.090^{*}$ & 0.0396 & $-0.813^{*}$ & 0.3587 \\
\hline Chronic medication (child) & $0.436^{*}$ & 0.1791 & 0.055 & 0.0383 & $0.648^{*}$ & 0.3145 \\
\hline Cohabitation status & 0.217 & 0.1904 & 0.016 & 0.0404 & 0.538 & 0.3561 \\
\hline Disability (parent) & -0.361 & 0.2062 & $-0.106^{*}$ & 0.0438 & -0.514 & 0.4090 \\
\hline Educational level & $-0.137^{*}$ & 0.0677 & $-0.062^{* * *}$ & 0.0144 & $-0.592^{* * *}$ & 0.1307 \\
\hline Employment status & -0.135 & 0.1677 & -0.023 & 0.0356 & 0.273 & 0.3201 \\
\hline
\end{tabular}

SE, standard error.

${ }^{1}$ Interaction $=$ (age group $) *$ (private health insurance) .

${ }^{*} p<0.05,{ }^{* *} p<0.01,{ }^{* *} p<0.001$.

In addition, outpatient costs differed significantly according to all variables with the exception of employment status, and the frequency of outpatient visits differed significantly according to age group, chronic medication in children, children's disability status, cohabitation, and parental disability status.

\section{Effects of children's private health insurance enrolment on inpatient care utilization and costs}

Table 5 shows the results of the analysis of the effects of loglevel inpatient costs, admission frequency, and the number of inpatient days in the fixed effects model. These results can be interpreted as follows:

First, inpatient costs, admission frequency, and the annual number of inpatient days differed significantly according to age group. Inpatient care utilization and costs decreased when children moved from the 1 to 5 years age group to the 6 to 10 years age group. Similar to outpatient care, this could be explained by a decrease in healthcare utilization due to an increase in age. Second, PHI enrolment resulted in an increase in inpatient costs and admission frequency but did not exert a 
significant influence on the number of inpatient days. This finding is consistent with those of previous research and is ascribable to the fact that the durations of hospital stays are seldom influenced by patients' decisions. However, in healthcare areas that were not covered by the NHI plan, $\mathrm{PHI}$ enrollees' inpatient costs showed a greater increase. Third, the interaction between insurance and age group was nonsignificant with respect to inpatient care utilization and costs. This indicates that external factors, including PHI and policies, did not exert a noticeable influence on inpatient care.

In summary, unlike the logit model, in which child health status did not exert a significant influence on variables related to children's PHI enrolment, child healthcare utilization was strongly influenced by health-related factors such as health and disability status. This could be considered to support the hypothesis that children's PHI enrolment depends upon parental SES.

In particular, PHI resulted in increases in both outpatient and inpatient costs and, along with other SES factors such as household income and parental educational level, exerted a strong influence on children's access to healthcare.

Although children were divided into two age groups to examine the effects of the copayment waiver policy for children younger than 6 years of age, it was difficult to differentiate between the age-dependent decreases in healthcare utilization due to economic reason and those resulting from physical growth. Both outpatient and inpatient healthcare utilization and costs decreased as children moved from the 1 to 5 years age group to the 6 to 10 years age group. The results were similar when children's individual ages, rather than age groups, were used. Only the interaction between age group and PHI enrolment exerted a significant effect on outpatient costs, and health expenditure increased, while healthcare utilization decreased. This suggests that even though healthcare utilization decreased with increasing age, PHI enrolment led to increases in outpatient costs.

\section{DISCUSSION}

In this study, 4-year Korea Health Panel data were used to examine the factors determining children's PHI enrolment decisions and the effects of $\mathrm{PHI}$ enrolment on pediatric healthcare utilization and costs. In Korea, PHI has gained a foothold as supplemental health insurance, and the $\mathrm{PHI}$ enrolment rate for children younger than 15 years of age was $85 \%$ according to the 2011 Health Panel. However, children's PHI is subject to health inequity, in that it is decided according to parental SES, irrespective of children's health status or willingness, and this was evident in the present study.

In the logit model based on the 2011 cross-sectional analysis, PHI enrolment status was used as an independent variable, and related child-specific and parent-specific individual and socioeconomic variables were examined. As expected, children's PHI enrolment was strongly associated with parental SES. Children's PHI enrolment was not influenced by children's health-related variables (i.e., medication taken for 3 months or longer and disability status), but it was significantly negatively influenced by parental disability status. In addition, parental educational level, working status, and household income quintile exerted a significant influence on children's PHI enrolment, particularly in low-SES groups. As PHI enrolment was included in cross-sectional analysis of data for only one year, unlike the panel analysis of healthcare utilization discussed below, interpretation of the results was limited.

In the fixed effects model in the panel analysis, annual panel data from 2008 to 2011 were used to analyze the effects of children's PHI enrolment on the outpatient and inpatient domains of healthcare utilization. As panel data involves repeated use of the same panel group, autocorrelation and concurrent correlation between individuals occurs. Moreover, because this study did not consider family relationships separately, sibling correlations between panel individuals also occurred, leading to omitted-variable bias due to individual characteristics involving health status or healthcare utilization tendencies. These issues were addressed using a panel fixed effects model in which error terms that influenced healthcare utilization were estimated as individual units.

In contrast to the factors determining PHI enrolment, children's individual variables, health status, and disability status exerted a strong influence on healthcare utilization in the panel model for the effects of children's PHI on their healthcare utilization. While it is natural that children's health status influenced their healthcare utilization, the influence of socioeconomic factors, such as parental educational level, household income, and cohabitation, should be not overlooked. In particular, the influence of children's PHI enrolment on healthcare costs was stronger relative to that of healthcare utilization frequency. Considering this in terms of the quantity and quality of healthcare utilization, low levels of parental SES could result in lower healthcare utilization quality for similar health- 
care utilization levels.

In the comparison between outpatient and inpatient care in the panel model, both outpatient and inpatient costs increased significantly with children's PHI enrolment. The frequency of outpatient visits and the total number of inpatient days were not significantly influenced by PHI enrolment, presumably because the durations of hospital stays are usually decided by physicians.

Previous studies have presented varying effects of socioeconomic factors, such as poverty, on children. Children from lowincome families show high accident and morbidity rates including those for neonatal death, low birthweight, and healthcare utilization [5]. Moreover, childhood poverty affects their cognitive development and affective states, and lowers the likelihood of opportunities for higher education. It also exerts a broad range of effects on adolescent risk behavior, peer relationships, and obesity levels [33]. An ecological study that compared a large number of countries reported that individuals were more vulnerable to childhood poverty in countries with a high degree of inequity [34]. Although the results of these studies did not elucidate the direct effects of $\mathrm{PHI}$ on health, different patterns of healthcare utilization were observed between children with and without PHI. This indicates that PHI enrolment was directly and indirectly associated with socioeconomic factors that exerted an influence on healthcare.

Socioeconomic factors include not only income level but also human, material, and social resources and the numerous tangible and intangible factors derived from them. A number of indicators, such as minimum cost of living, relative poverty threshold using median income, and asset level, are used to define poverty [35]. While no poverty threshold was established in the present study, household income quintile was used to determine the link between income level and PHI enrolment, and socioeconomic factors were determined using additional information such as educational level. When insurance enrollees and non-enrollees were compared in a descriptive statistical analysis, the non-enrollees exhibited lower educational levels (Table 2). However, socioeconomic factors cannot fully explain insurance enrolment decisions, which can vary according to individual tendencies or circumstances. For example, individuals with high levels of income could decide against insurance enrolment because of low expectations regarding efficiency, and individuals with low levels of income could purchase insurance with the aid of relatives, even under financial strain.
In contrast, in considering adult insurance enrolment, problems associated with adverse selection, moral hazard, and cream-skimming should be taken into account, provided that sufficient detail concerning the individual's health status is available. However, in our analysis, children's health status did not exert a significant influence on decisions regarding insurance enrolment; therefore, these problems do not exist in the children's insurance market. For this reason, exclusion from insurance enrolment because of being considered high risk and insurance enrolment with the intention of using intensive healthcare can be ruled out. Consequently, insurance enrolment decisions are strongly influenced by SES. The results of the analysis showed that outpatient and inpatient health spending increased following insurance enrolment. While this could be interpreted as moral hazard, it could also be considered insufficient healthcare utilization quality in PHI non-enrollees. Considering the fact that PHI enrolment does not exert any noticeable influence on outpatient or inpatient healthcare consumption, the majority of children are PHI enrollees, and PHI plays a supplementary role, unmet healthcare needs are likely to increase for SES groups for which PHI is beyond reach.

This study's contribution to PHI research can be summarized as follows: First, this was the first Korean study to examine PHI enrolment factors and analyze the effects of PHI enrolment on healthcare utilization, focusing on pediatric insurance enrollees. As a result, children's insurance enrolment factors were closely associated with parental socioeconomic factors. The results of the comparison of healthcare utilization patterns between PHI enrollees and non-enrollees revealed that PHI enrolment led to increased healthcare expenditure. As the frequency of healthcare utilization and the number of inpatient days did not differ significantly between these groups, such differences could be regarded as related to the quality, rather than the quantity of healthcare utilization. In addition, increases in healthcare expenditure following insurance enrolment, in the absence of cream skimming and reverse selection, could be interpreted as either partial moral hazard or insufficient healthcare expenditure in the absence of insurance enrolment.

With respect to the limitations of the study, the age data were inaccurate, as the Health Panel provided only the year of birth, without exact dates. Consequently, changes in healthcare utilization could not be estimated precisely because of the lack of exact time points for the shift from the 1 to 5 years age group to the 6 to 10 years age group. The same problem was encountered for infants younger than 1 year of age. As 
the distinction between the ages of 0 and 1 year changed according to survey time points, the former was integrated into the latter. In the panel model, children were divided into two age groups, to determine the effects of the copayment waiver policy for children younger than 6 years of age. However, given that negative values were shown in all models, additional research is required to determine whether the decrease in healthcare utilization occurred because of the copayment waiver policy or children's natural development. The interaction between PHI enrolment and age group exerted a significant effect only in the outpatient cost model. The finding that outpatient healthcare costs increased, even with a reduction in the frequency of outpatient visits, is indicative of a strong impact of PHI enrolment on outpatient costs.

Another inaccuracy concerned changes, such as cancellation, in insurance enrolment status in $2 \%$ to $3 \%$ of enrollees. In such cases, if insurance plans were changed or enrollees held more than one insurance policy, they were processed as $\mathrm{PHI}$ enrollees. Changes in insurance type were not accounted for in this process, because no type-dependent differentiation (e.g., between indemnity and fixed-amount types) was made in this study. This factor should be examined in further studies.

Furthermore, $\mathrm{PHI}$ claims and the resultant reimbursement amounts were not included in the analysis. To estimate the overall economic effects of $\mathrm{PHI}$ enrolment on households, insurance premiums, healthcare costs, and reimbursement amounts should be included in analyses. It would be desirable to examine with this as a separate topic.

\section{CONFLICT OF INTEREST}

The authors have no conflicts of interest associated with the material presented in this paper.

\section{REFERENCES}

1. Kim CY. Theories of health security. Paju: Hanul Publishing; 2009, p. 16-99 (Korean).

2. Kim HR, Kang YH, Yun KJ, Kim CS. Socioeconomic health inequalities and counter policies in Korea. Seoul: Korea Institute for Health and Social Affairs; 2004, p. 57-81 (Korean).

3. Kim HR. Obesity and underweight among children in low income families: status, and policy options for childhood health equality. Health Welf Policy Forum 2012;(188):55-66 (Korean).

4. Aber JL, Bennett NG, Conley DC, Li J. The effects of poverty on child health and development. Annu Rev Public Health 1997; 18:463-483.

5. Kwon ES, Ku IH. The effect of poverty on children's health. Korean J Soc Welf 2010;62(4):129-148 (Korean).

6. Lee BJ, Kim SS, Kim NH. An exploratory study on Korean children's development: the effects of socioeconomic factors and social capital. J Korean Soc Child Welf 2010;13(2):107-141 (Korean).

7. Organization for Economic Cooperation and Development (OECD). OECD health care quality review: Korea assessment and recommendations; 2012 [cited 2015 Oct 10]. Available from: http://www.oecd.org/els/health-systems/49818570.pdf.

8. Choi WH. The impact of inpatient copayment waiver on medical service utilization of children under 6 [dissertation]. Seoul: Seoul National University; 2008 (Korean).

9. Jung KT. A study on private health insurance in Korea. Korean J Health Policy Adm 1997;7(2):109-146 (Korean).

10. Freeman JD, Kadiyala S, Bell JF, Martin DP. The causal effect of health insurance on utilization and outcomes in adults: a systematic review of US studies. Med Care 2008;46(10):1023-1032.

11. Huh EJ, Kim JK. Middle-aged and old people's purchase and expenditure decision factors of private health insurance. J Consum Stud 2009;20(4):107-127 (Korean).

12. Kim DH, Lee BJ. An analysis on adverse selection in fee-for-service health insurance. Korean Insur J 2013;96:25-50 (Korean).

13. Lee HB, Hyun KR. A study for characteristics and factors of private health insurers. Korean Soc Secur Stud 2011;27(1):217240 (Korean).

14. Lee YC, Im BH, Park YH. The determinants and comparison of health behavior and health service by private medical insurance on National Health-Nutrition Survey. J Korea Contents Assoc 2010;10(12):190-204 (Korean).

15. Park SB, Jeong KH. The determinants of private insurance and its effects on medical utilization in Korea. Korean Insur J 2011; 88:23-49 (Korean).

16. Baek IR, Byun SS, Park H. A study on joining private health insurance of the socially vulnerable and medical service utilization of private insurance members. Health Soc Sci 2012;31: 127-151 (Korean).

17. Baek IR, Park H, Byun SS. The determinants and medical care utilization behavior of private health insurance. J Korea Contents Assoc 2012;12(7):295-305 (Korean).

18. Jeon BY, Kwon SM. The effect of health care expenditure on the change in the demand for private health insurance in Korea. Korean J Health Econ Policy 2012;18(4):65-84 (Korean). 
19. Kwon HJ. Effects of economic factor on membership transition in private health insurance. Soc Welf Policy 2011;38(3): 131-158 (Korean).

20. Yun HS. Effects of private insurance on medical expenditure. KDI J Econ Policy 2008;30(2):99-128 (Korean).

21. Kang SW, You CH, Oh EH, Kwon YD. The impact of having private health insurance on healthcare utilization with controlling for endogeneity. Korean J Health Econ Policy 2010;16(1): 139-159 (Korean).

22. Kim DH. Effects of fee-for-service health insurance on medical consumption. Korean Insur J 2014;98:61-90 (Korean).

23. Lee CW. The effect of private health insurance on medical use. Korean J Health Econ Policy 2010;16(2):1-15 (Korean).

24. Kim SM, Kwon YD. Private health insurance and the use of health care services: a review of empirical research in Korea. Korean J Health Serv Manag 2011;5(4):177-192 (Korean).

25. Jung YH. Fact about the private health insurance enrollment according to Korea Health Panel. Issue Focus 2011;(70):1-8 (Korean).

26. Newacheck PW, Stoddard JJ, Hughes DC, Pearl M. Health insurance and access to primary care for children. N Engl J Med 1998;338(8):513-519.

27. Olson LM, Tang SF, Newacheck PW. Children in the United States with discontinuous health insurance coverage. N Engl J Med 2005;353(4):382-391.

28. Szilagyi PG, Dick AW, Klein JD, Shone LP, Zwanziger J, Mcln- erny T. Improved access and quality of care after enrollment in the New York State Children's Health Insurance Program (SCHIP). Pediatrics 2004;113(5):e395-e404.

29. Victora CG, Wagstaff A, Schellenberg JA, Gwatkin D, Claeson M, Habicht JP. Applying an equity lens to child health and mortality: more of the same is not enough. Lancet 2003;362(9379): 233-241.

30. You CH, Kang SW, Choi JH, Oh EH, Kwon YD. The effect of private health insurance on health care utilization: evidence from Korea Health Panel (2008-2010). Korean J Health Serv Manag 2014;8(2):101-113 (Korean).

31. Rothschild M, Stiglitz J. Equilibrium in competitive insurance markets: an essay on the economics of imperfect information. Quart J Econ 1976;90(4):629-649.

32. Wooldridge JM. Introductory econometrics: a modern approach. 5th ed. Mason: South-Western Cengage Learning; 2012, p. 484-500.

33. Hackman DA, Farah MJ. Socioeconomic status and the developing brain. Trends Cogn Sci 2009;13(2):65-73.

34. Pickett KE, Wilkinson RG. Child wellbeing and income inequality in rich societies: ecological cross sectional study. BMJ 2007; 335(7629):1080.

35. Wagstaff A, Watanabe N. What difference does the choice of SES make in health inequality measurement? Health Econ 2003;12(10):885-890. 